\title{
A CONVEX BODY ASSOCIATED TO THE BUSEMANN RANDOM SIMPLEX INEQUALITY AND THE PETTY CONJECTURE
}

\author{
J. HADDAD
}

\begin{abstract}
Given $L$ a convex body, the $L_{p}$-Busemann Random Simplex Inequality is closely related to the centroid body $\Gamma_{p} L$ for $p=1$ and 2 , and only in these cases it can be proved using the $L_{p}$-Busemann-Petty centroid inequality. We define a convex body $N_{p} L$ and prove an isoperimetric inequality for $\left(N_{p} L\right)^{\circ}$ that is equivalent to the $L_{p}$-Busemann Random Simplex Inequality. As applications, we give a simple proof of a general functional version of the Busemann Random Simplex Inequality and study a dual theory related to Petty's conjectured inequality. More precisely, we prove dual versions of the $L_{p}$-Busemann Random Simplex Inequality for sets and functions by means of the $p$-affine surface area measure, and we prove that the Petty conjecture is equivalent to an $L_{1}$-Sharp Affine Sobolev-type inequality that is stronger than (and directly implies) the Sobolev-Zhang inequality.
\end{abstract}

\section{INTRODUCTION}

Let $L \subseteq \mathbb{R}^{n}$ be a convex body (a compact convex set with non-empty interior) with the origin as interior point. In 1953 Busemann showed [2] that if $n$ points are chosen randomly inside $L$ with uniform probability, then the expected volume of the simplex formed by the convex hull of these points and the origin attains its minimum among all convex sets of the same volume, when $L$ is an ellipsoid. More precisely, if we denote

$$
D_{n}\left(v_{1}, \ldots, v_{n}\right)=\left|\operatorname{det}\left(v_{1}, \ldots, v_{n}\right)\right|,
$$

then $D_{n}\left(v_{1}, \ldots, v_{n}\right)$ is the volume of the parallelepiped spanned by the vectors $v_{1}, \ldots, v_{n}$. We have $\frac{1}{n !} D_{n}\left(v_{1}, \ldots, v_{n}\right)=\operatorname{vol}\left(\operatorname{co}\left(0, v_{1}, \ldots, v_{n}\right)\right)$ and the result can be stated as

$$
\int_{L} \cdots \int_{L} D_{n}\left(x_{1}, \ldots, x_{n}\right) d x_{1} \cdots d x_{n} \geq b_{n} \operatorname{vol}(L)^{n+1}
$$

where $b_{n}$ is a sharp constant, and equality holds if and only if $L$ is an origin-symmetric ellipsoid. This is known as the Busemann Random Simplex Inequality.

A convex body may be characterized by its support function $h_{K}$, defined as

$$
h_{K}(y)=\max \{\langle y, z\rangle: z \in K\} .
$$

It describes the (signed) distance of supporting hyperplanes of $K$ to the origin. Closely related to inequality (11), the centroid body of $L$ is defined as the unique convex body $\Gamma_{1} L$ having as support function

$$
h\left(\Gamma_{1} L, \xi\right)=\frac{1}{c_{n, 1} \operatorname{vol}(L)} \int_{L}|\langle\xi, x\rangle| d x
$$

where the constant $c_{n, 1}$ is such that if $\mathbb{B}_{n}$ is the unit euclidean ball, $\Gamma_{1} \mathbb{B}_{n}=\mathbb{B}_{n}$.

It is not difficult to show (see [26], formulas 10.69 and 5.82) that

$$
\operatorname{vol}\left(\Gamma_{1} L\right)=c_{n, 1}^{-n} \frac{2^{n}}{n !} \operatorname{vol}(L)^{-n} \int_{L} \cdots \int_{L} D_{n}\left(x_{1}, \ldots, x_{n}\right) d x_{1} \cdots d x_{n}
$$

2000 Mathematics Subject Classification: Primary 52A22, Secondary 46E35

Keywords: Convex Body, Busemann Random Simplex inequality, Petty conjecture, Sobolev inequality 
and inequality (1) is equivalent to the well known Busemann-Petty centroid inequality

$$
\operatorname{vol}\left(\Gamma_{1} L\right) \geq \operatorname{vol}(L),
$$

where equality holds if and only if $L$ is an origin-symmetric ellipsoid.

Several generalizations and extensions of inequality (11) have been studied. Groemer proved in [9] that the expected value, as well as the higher order moments of the volume of the convex hull of $n+1$ points inside $K$ is minimized when $K$ is an ellipsoid. Then in [10] extended the result to the case where the number of points $k$ is allowed to be greater than or equal to $n+1$. Pfiefer 24] extended the result to measurable sets, and were the volume is composed with an increasing function. Hartzoulaki and Paouris [13] proved it replacing the volume by the Quermassintegrals. The reverse inequality, with triangles as equality cases (in the planar case) was studied by Campi, Colesanti and Gronchi [5, 4 and Saroglou 25. Dann, Paouris and Pivovarov in [7] showed functional inequalities for any number of points $k \geq 1$, and Paouris and Pivovarov in [22] showed a general condition allowing to apply Steiner symmetrization to many isoperimetric inequalities in stochastic form.

A general version of inequality (1) where the $k$ bodies in the multiple integral are allowed to be different, and the volume of the parallelepiped is raised to the power $p>0$, was proved in [1]. We state here a particular case.

Theorem 1.1. Let $L_{1}, \ldots, L_{n}$ be convex bodies and $p \geq 1$, define

$$
I_{p}\left(L_{1}, \ldots, L_{n}\right)=\int_{L_{1}} \cdots \int_{L_{n}} D_{n}\left(x_{1}, \ldots, x_{n}\right)^{p} d x_{1} \cdots d x_{n}
$$

then we have

$$
I_{p}\left(L_{1}, \ldots, L_{n}\right) \geq b_{n, p} \prod_{i=1}^{n} \operatorname{vol}\left(L_{i}\right)^{\frac{n+p}{n}}
$$

where $b_{n, p}$ is such that equality holds if and only if $L_{1}, \ldots, L_{n}$ are homothetic origin-symmetric ellipsoids. We refer to [27, Theorem 8.6.1] for the explicit value of $b_{n, p}$.

It is important to mention that the proofs of these theorems are all based on some form of Steiner symmetrization. We propose a different approach based on the construction of a convex body $N_{p}\left(L_{1}, \ldots, L_{n-1}\right)$. In [19, 17] Lutwak, Yang and Zhang defined the $L_{p}$ Centroid body $\Gamma_{p} L$ and proved an $L_{p}$ version of the Busemann-Petty centroid inequality

$$
\operatorname{vol}\left(\Gamma_{p} L\right) \geq \operatorname{vol}(L),
$$

where equality holds if and only if $L$ is an origin-symmetric ellipsoid. The body $\Gamma_{p} L$ is defined by the support function

$$
h\left(\Gamma_{p} L, \xi\right)^{p}=\frac{1}{c_{n, p} \operatorname{vol}(L)} \int_{L}|\langle\xi, x\rangle|^{p} d x
$$

where $c_{n, p}$ is such that $\Gamma_{p} \mathbb{B}_{n}=\mathbb{B}_{n}$. In general, it doesn't seem to exist any relation between inequalities (3) and (2), except for $p=1$ and $p=2$, (see [4, p. 4]). The main purpose of this paper is to construct a convex body containing the information of the $L_{p}$-Busemann Random Simplex Inequality, playing the role of the centroid body for $p \geq 1$, and obtain some inequalities related to it. The inequalities we obtain are already known to be true in some particular cases, but the method is new and very simple, no symmetrization arguments are needed. The second objective is to study a dualization of Theorem 1.1 that is suggested by the proof of Theorem 1.5 below.

We start with the (fairly obvious) definition. 
Definition 1.2. Let $L_{1}, \ldots, L_{n-1}$ be convex bodies, we define $N_{p}\left(L_{1}, \ldots, L_{n-1}\right)$ as the convex body defined by the support function

$$
h\left(N_{p}\left(L_{1}, \ldots, L_{n-1}\right), \xi\right)^{p}=\int_{L_{1}} \cdots \int_{L_{n-1}} D_{n}\left(x_{1}, \ldots, x_{n-1}, \xi\right)^{p} d x_{1} \cdots d x_{n-1} .
$$

We write $N_{p} L=N_{p}(L, \ldots, L)$.

The polar of a convex body $K$ is the convex body defined by

$$
K^{\circ}=\left\{x \in \mathbb{R}^{n} / h(K, x) \leq 1\right\} .
$$

We shall prove the following

Theorem 1.3. Let $L_{1}, \ldots, L_{n-1}$ be convex bodies, then

$$
\operatorname{vol}\left(N_{p}^{\circ}\left(L_{1}, \ldots, L_{n-1}\right)\right) \leq a_{n, p} \prod_{i=1}^{n-1} \operatorname{vol}\left(L_{i}\right)^{-\frac{n+p}{p}},
$$

where $a_{n, p}=\left(\frac{n+p}{n} b_{n, p}\right)^{-\frac{n}{p}}$ and $N_{p}^{\circ}\left(L_{1}, \ldots, L_{n-1}\right)$ stands for the polar body of $N_{p}\left(L_{1}, \ldots, L_{n-1}\right)$.

The inequality is invariant under volume-preserving affine transformations. Also, equality holds in (4) if and only if $L_{1}, \ldots, L_{n-1}$ are homothetic origin-symmetric ellipsoids. Moreover, Theorem 1.3 is equivalent to Theorem 1.1, and the only tool we use to prove this equivalence is the dual mixed volume inequality. Let us define for two convex bodies $K, L$, the dual mixed volume as

$$
\tilde{V}_{-p}(K, L)=\frac{n+p}{n} \int_{K} h_{L^{\circ}}(x)^{p} d x .
$$

The dual mixed volume inequality states that $\tilde{V}_{-p}(K, L) \geq \operatorname{vol}(K)^{\frac{n+p}{n}} \operatorname{vol}(L)^{-\frac{p}{n}}$. Then it suffices to note that

$$
I_{p}\left(L_{1}, \ldots, L_{n}\right)=\frac{n}{n+p} \tilde{V}_{-p}\left(L_{n}, N_{p}^{\circ}\left(L_{1}, \ldots, L_{n-1}\right)\right) .
$$

If we know Theorem 1.3 to be true we obtain

$$
I_{p}\left(L_{1}, \ldots, L_{n}\right) \geq \frac{n}{n+p} \operatorname{vol}\left(L_{n}\right)^{\frac{n+p}{n}} \operatorname{vol}\left(N_{p}^{\circ}\left(L_{1}, \ldots, L_{n-1}\right)\right)^{-\frac{p}{n}} \geq b_{n, p} \operatorname{vol}\left(L_{n}\right)^{\frac{n+p}{n}} \prod_{i=1}^{n-1} \operatorname{vol}\left(L_{i}\right)^{\frac{n+p}{n}} .
$$

Conversely, if we know Theorem 1.1 to be true, we can compute

$$
\begin{aligned}
\operatorname{vol}\left(N_{p}^{\circ}\left(L_{1}, \ldots, L_{n-1}\right)\right) & =\tilde{V}_{-p}\left(N_{p}^{\circ}\left(L_{1}, \ldots, L_{n-1}\right), N_{p}^{\circ}\left(L_{1}, \ldots, L_{n-1}\right)\right) \\
& =\frac{n+p}{n} I_{p}\left(L_{1}, \ldots, L_{n-1}, N_{p}^{\circ}\left(L_{1}, \ldots, L_{n-1}\right)\right) \\
& \geq \frac{n+p}{n} b_{n, p} \operatorname{vol}\left(N_{p}^{\circ}\left(L_{1}, \ldots, L_{n-1}\right)\right)^{\frac{n+p}{n}} \prod_{i=1}^{n-1} \operatorname{vol}\left(L_{i}\right)^{\frac{n+p}{n}},
\end{aligned}
$$

and obtain Theorem 1.3 .

The definition of $N_{p}\left(L_{1}, \ldots, L_{n-1}\right)$ fits perfectly to prove a functional version of inequality (2). Let $l_{1}, \ldots, l_{n-1}: \mathbb{R}^{n} \rightarrow \mathbb{R}$ be continuous non-negative functions with compact support, and let us define

$$
I_{p}\left(l_{1}, \ldots, l_{n}\right)=\int_{\mathbb{R}^{n}} \cdots \int_{\mathbb{R}^{n}} l_{1}\left(x_{1}\right) \cdots l_{n}\left(x_{n}\right) D_{n}\left(x_{1}, \ldots, x_{n}\right)^{p} d x_{1} \cdots d x_{n} .
$$

Notice that we recover the previous definition of $I_{p}$ if we take the functions $l_{i}$ to be indicator functions of convex bodies. We define accordingly the set $N_{p}\left(l_{1}, \ldots, l_{n-1}\right)$. 
Definition 1.4. Let $l_{1}, \ldots, l_{n-1}$ be continuous non-negative functions with compact support, we define $N_{p}\left(l_{1}, \ldots, l_{n-1}\right)$ as the convex body defined by the support function

$$
h\left(N_{p}\left(l_{1}, \ldots, l_{n-1}\right), \xi\right)^{p}=\int_{\mathbb{R}^{n}} \cdots \int_{\mathbb{R}^{n}} l_{1}\left(x_{1}\right) \cdots l_{n-1}\left(x_{n-1}\right) D_{n}\left(x_{1}, \ldots, x_{n-1}, \xi\right)^{p} d x_{1} \cdots d x_{n-1} .
$$

The subject of functional inequalities with geometric counterpart attracted great interest in recent years (see for example [8, 3, 11]), specially affine invariant functional inequalities, that often imply an euclidean version. For this reason we propose to study several extensions of our results to the functional setting.

Let us define the function

$$
G_{p, \lambda}(s)=\left\{\begin{array}{cc}
\left(1+|s|^{p}\right)^{1 /(\lambda-1)}, & \lambda<1 \\
\left(1-|s|^{p}\right)_{+}^{1 /(\lambda-1)}, & \lambda>1 \\
\chi_{[-1,1]}(s), & \lambda=+\infty .
\end{array}\right.
$$

Here $t_{+}=\max \{t, 0\}$ and $\chi_{[-1,1]}$ denotes the characteristic function of the set $[-1,1]$.

Theorem 1.5. Let $l_{1}, \ldots, l_{n-1}$ be continuous non-negative functions with compact support, and take any $\lambda \in\left(\frac{n}{n+p}, 1\right) \cup(1,+\infty]$. Then,

$$
\operatorname{vol}\left(N_{p}^{\circ}\left(l_{1}, \ldots, l_{n-1}\right)\right) \leq A_{n, p, \lambda} \prod_{i=1}^{n-1}\left\|l_{i}\right\|_{1}^{-\frac{n+p \lambda^{\prime}}{p}}\left\|l_{i}\right\|_{\lambda}^{\lambda^{\prime}}
$$

where $A_{n, p, \lambda}$ is a sharp constant and equality holds if and only if the functions $l_{i}$ have the form $l_{i}(x)=$ $a_{i} G_{p, \lambda}\left(\left|b_{i} A .\left(x-x_{i}\right)\right|\right)$ with $x_{0} \in \mathbb{R}^{n}, a_{i}, b_{i}>0$ and $A \in \mathrm{Gl}_{n}(\mathbb{R})$. The explicit value of $A_{n, p, \lambda}$ is computed in the proof of Theorem.

The equivalence between Theorems 1.1 and 1.3 remains valid in the functional setting and we obtain

Corollary 1.6. Let $l_{1}, \ldots, l_{n}$ be continuous non-negative functions with compact support, and take any $\lambda \in\left(\frac{n}{n+p}, 1\right) \cup(1,+\infty]$. Then,

$$
I_{p}\left(l_{1}, \ldots, l_{n}\right) \geq B_{n, p, \lambda} \prod_{i=1}^{n}\left\|l_{i}\right\|_{1}^{\frac{n+p \lambda^{\prime}}{n}}\left\|l_{i}\right\|_{\lambda}^{-\frac{p \lambda^{\prime}}{n}}
$$

and equality holds if and only if the functions $l_{i}$ are extremal functions of Theorem 1.5. The explicit value of $B_{n, p, \lambda}$ is computed in the proof of the Corollary.

The case $\lambda=\infty$ of Corollary 1.6 appears already in [22], application number 10, Section 5. A more general version where the number of vertices in the random simplex is $k \leq n$ appears in [7, Corollary 4.2]. Throughout this paper the operator $D_{n}$ can be replaced by any positive function being homogeneous of degree 1 in each variable, and any number of variables. Considering for example $D_{k}\left(v_{1}, \ldots, v_{k}\right)$ defined as the $k$ dimensional volume of the parallelepiped spanned by the vectors $v_{1}, \ldots, v_{k}$, since [7, Corollary 4.2] proves Theorem 1.1 for $k \leq n$ points, then the equivalence between Theorem 1.1 and Corollary 1.6 applies, and we recover a general version of [7, Corollary 4.2] for $\lambda \in\left(\frac{n}{n+p}, 1\right) \cup(1,+\infty]$.

The proof of Theorem 1.5] uses a moment inequality (Lemma 2.1) proved in [18] (this is a dual mixed volume inequality for functions), and an induction argument. Given the simplicity of the proofs of Theorem 1.5 and Corollary [1.6, we propose to study a dual random process and an associated convex set $\tilde{N}_{p}\left(L_{1}, \ldots, L_{n-1}\right)$. The motivation is the following: since all the tools involved in the proofs of Theorem 1.5 and Corollary 1.6 have dual versions (the dual mixed volume and the dual mixed volume inequality), we can also prove an equivalence between a volume inequality for the set $\tilde{N}_{p}\left(L_{1}, \ldots, L_{n-1}\right)$ and an inequality 
for a dual functional $\tilde{I}_{p}\left(L_{1}, \ldots, L_{n}\right)$ by means of the mixed volume and the mixed volume inequality. This way we arrive to what we believe should be the correct dualization of $I_{p}\left(L_{1}, \ldots, L_{n}\right)$.

Definition 1.7. Let $L_{1}, \ldots, L_{n}$ be convex bodies and $l_{1}, \ldots, l_{n}$ be continuous non-negative functions with compact support, define

$$
\begin{aligned}
\tilde{I}_{p}\left(L_{1}, \ldots, L_{n}\right) & =\int_{S^{n-1}} \cdots \int_{S^{n-1}} D_{n}\left(\xi_{1}, \ldots, \xi_{n}\right)^{p} d S_{p, L_{1}}\left(\xi_{1}\right) \ldots d S_{p, L_{n}}\left(\xi_{n}\right) \\
\tilde{I}_{p}\left(l_{1}, \ldots, l_{n}\right) & =\int_{\mathbb{R}^{n}} \cdots \int_{\mathbb{R}^{n}} D_{n}\left(\nabla l_{1}\left(x_{1}\right), \ldots, \nabla l_{n}\left(x_{n}\right)\right)^{p} d x_{1} \cdots d x_{n}
\end{aligned}
$$

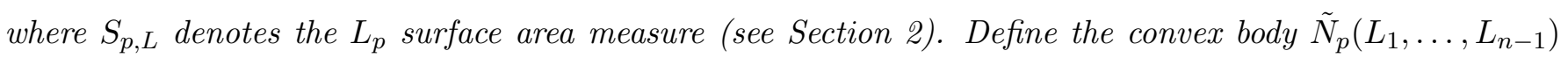
by the support function

$$
h\left(\tilde{N}_{p}\left(L_{1}, \ldots, L_{n-1}\right), \xi\right)^{p}=\int_{S^{n-1}} \ldots \int_{S^{n-1}} D_{n}\left(\xi_{1}, \ldots, \xi_{n-1}, \xi\right)^{p} d S_{p, L_{1}}\left(\xi_{1}\right) \ldots d S_{p, L_{n-1}}\left(\xi_{n-1}\right)
$$

The quantity $S_{p, L}(L)^{-n} \tilde{I}_{p}(L, \ldots, L)$ represents the expected $p$-th power of the volume of a random parallelepiped generated by the normal vectors $n_{x_{1}}, \cdots, n_{x_{n}}$ at points $x_{i}$ chosen randomly in the surface of $L$, with probability measure $S_{p, L} / S_{p, L}(L)$. The definition of $\tilde{I}_{p}\left(l_{1}, \ldots, l_{n}\right)$ is based on the definition of the surface area measure of a function (see Section 2), and for $p=1$ can be interpreted in the weak sense if $l_{1}, \ldots, l_{n}$ are indicator functions of convex bodies.

The inequalities that should hold with respect to $\tilde{I}_{p}$ and $\tilde{N}_{p}$ in order to dualize the proof of Theorem 1.5 are summarized in Section 5 but they are open problems. Let us mention the following: for $1 \leq p<n$ we ask if the inequality

$$
\tilde{I}_{p}\left(L_{1}, \ldots, L_{n}\right) \geq \bar{b}_{n, p} \prod_{i=1}^{n} \operatorname{vol}\left(L_{i}\right)^{\frac{n-p}{n}}
$$

holds, where the sharp constant is such that equality holds in (5) if and only if $L_{1}, \ldots, L_{n}$ are homothetic origin-symmetric ellipsoids for $p>1$ and homothetic ellipsoids for $p=1$. Again, the case $p=1$ is special. The projection body of a convex body $K$ is defined by the support function

$$
h(\Pi K, \xi)=\frac{1}{2} \int_{S^{n-1}}|\langle\xi, \eta\rangle| d S_{K}(\eta)
$$

and it is easy to see that $\operatorname{vol}(\Pi K)=\frac{1}{n !} \tilde{I}_{1}(K, \ldots, K)$. Then inequality (5) with $p=1$ and $L_{1}=\cdots=L_{n}=L$ is Petty's conjectured inequality, one of the major open problems in Convex Geometry. This is that

$$
\operatorname{vol}(L)^{1-n} \operatorname{vol}(\Pi L) \geq\left(\frac{\omega_{n-1}}{\omega_{n}}\right)^{n} \omega_{n}^{2}
$$

where equality holds if and only if $L$ is an origin-symmetric ellipsoid. Also, as a consequence of the Aleksandrov-Fenchel inequality (see [14, (1.7)] or [26, (7.64)] for the proof), we have

$$
\frac{1}{n !} \tilde{I}_{1}\left(L_{1}, \ldots, L_{n}\right)=V\left(\Pi L_{1}, \ldots, \Pi L_{n}\right) \geq \prod_{i=1}^{n} \operatorname{vol}\left(\Pi L_{i}\right)^{1 / n}
$$

so (6) is also equivalent to (5) with $p=1$ and arbitrary convex bodies $L_{1}, \ldots, L_{n}$. We refer to [26, Section 10.9] for an overview about recent developments and main difficulties around this conjecture. In Section 5 we 
shall prove that (5) for $p=1$ (and thus the Petty conjecture) implies the sharp, affine invariant Sobolev-like inequality

$$
\|f\|_{\frac{n}{n-1}} \leq C_{n}\left(\int_{\mathbb{R}^{n}} \cdots \int_{\mathbb{R}^{n}} D_{n}\left(\nabla f\left(x_{1}\right), \ldots, \nabla f\left(x_{n}\right)\right) d x_{1} \cdots d x_{n}\right)^{\frac{1}{n}}
$$

that is stronger than (and directly implies) the sharp affine Sobolev inequality proved by Zhang [28].

In Section 4 we prove a weaker result that is consequence of Corollary 1.6.

Theorem 1.8. Let $L_{1}, \ldots, L_{n}$ be convex bodies with $C^{2}$-smooth boundary and positive Gauss curvature, then

$$
\tilde{I}_{p}\left(L_{1}, \ldots, L_{n}\right) \geq \tilde{b}_{n, p} \prod_{i=1}^{n} \Omega_{p}\left(L_{i}\right)^{\frac{n+p}{n}}
$$

where $\tilde{b}_{n, p}=(n+p)^{n} \frac{b_{n, p}}{n^{n+p}}$ and $\Omega_{p}(L)$ denotes the $p$-affine surface area of $L$ and equality holds if and only if $L_{1}, \ldots, L_{n}$ are homothetic origin-symmetric ellipsoids.

Theorem 1.8 is weaker than inequality (5) and it falls short for polytopes since $\Omega_{p}\left(L_{i}\right)=0$ in that case. We believe Theorem 1.8 can be improved in this regard. Notice that for $p=1$ and $L_{1}=\cdots=L_{n}=L$ we recover Petty's affine projection inequality [23, see also [26, Section 10.9].

Finally, a functional version of Theorem 1.8 reads as follows.

Theorem 1.9. Let $l_{1}, \ldots, l_{n}$ be non-zero, $C^{2}$ smooth, non-negative functions with compact support. Assume additionally that $l_{1}, \ldots, l_{n}$ have convex level sets with positive Gauss curvature. Let $\alpha \in\left(\frac{n}{n+1}, 1\right) \cup(1, \infty)$, then

$$
\tilde{I}_{p}\left(l_{1}, \ldots, l_{n}\right) \geq \tilde{B}_{n, p, \alpha} \prod_{i=1}^{n}\left(\Omega_{p}\left(l_{i}\right)^{\frac{n+\alpha^{\prime}}{n+1}} \Omega_{p}\left(l_{i}^{\alpha}\right)^{-\frac{1}{n+1} \frac{1}{\alpha-1}}\right)^{\frac{n+p}{n}}
$$

where

$$
\Omega_{p}(l)=\int_{\mathbb{R}^{n}}|\operatorname{det}(K l(x))|^{\frac{p}{n+p}} d x, \text { and } K l(x)=\left(\begin{array}{c|c}
0 & \nabla l(x)^{T} \\
\hline \nabla l(x) & H l(x)
\end{array}\right)
$$

and with equality if each function $l_{i}$ has the form $l_{i}(x)=a_{i} F\left(b_{i}|A \cdot x|_{2}\right)_{+}$where $a_{i}, b_{i}>0, A \in \mathrm{Gl}_{n}(\mathbb{R})$ and $F:(0, T] \rightarrow[0,1)$ is a solution of the following ODE

$$
F^{\prime}(t)= \begin{cases}-t^{-\frac{n-1}{p-1}}\left(1-F(t)^{\frac{(\alpha-1)(n+1) p}{n+p}}\right)^{\frac{n+p}{p(p-1)}} & \text { if } \alpha>1 \\ -t^{-\frac{n-1}{p-1}}\left(F(t)^{\frac{(\alpha-1)(n+1) p}{n+p}}-1\right)^{\frac{n+p}{p(p-1)}} & \text { if } \alpha<1\end{cases}
$$

with $F(T)=0$. For $\alpha=\infty$ we obtain

$$
\tilde{I}_{p}\left(l_{1}, \ldots, l_{n}\right) \geq \tilde{B}_{n, p, \infty} \prod_{i=1}^{n} \Omega_{p}\left(l_{i}\right)^{\frac{n+p}{n}}\left\|l_{i}\right\|_{\infty}^{-\frac{p}{n}} .
$$

The explicit value of $\tilde{B}_{n, p, \alpha}$ is computed in the proof of the Theorem.

The quantity $\Omega_{p}(l)$ at the right-hand side can be interpreted as a functional version of the $p$-affine surface area measure, although other definitions can be found in the literature that are slightly different. For 
example, in formula (26) of [3], it is defined for a smooth convex function $\psi$ as

$$
\operatorname{as}_{\lambda}(\psi)=\int_{\mathbb{R}^{n}} \operatorname{det}(H \psi(x))^{\lambda} e^{-\psi(x)} d x
$$

It can be shown that if $l(x)=F\left(\|x\|_{K}\right)$ with $F:[0, \infty) \rightarrow \mathbb{R}_{+}$a $C^{2}$ smooth (not necessarily decreasing) function with compact support, then $\Omega_{p}(l)=\Omega_{p}(K)$ provided that $\int_{0}^{\infty} t^{\frac{n(n-1)}{n+p}}\left|F^{\prime}(t)\right|^{\frac{p(n+1)}{n+p}} d t=1$. We remark that in Theorem 3 of [3], a similar relation for $\operatorname{as}_{\lambda}(\psi)$ is proved only for $F(t)=\frac{t^{2}}{2}$. For the case $p=1$ and any $l(x)$ with convex level sets, our definition also satisfies

$$
\Omega_{1}(l)=\int_{0}^{\infty} \Omega_{1}\left(\left\{x \in \mathbb{R}^{n} / l(x) \geq t\right\}\right) d t .
$$

If $A \in \mathrm{Gl}_{n}(\mathbb{R})$ and $l_{A}(x)=l(A . x)$ then

$$
K l_{A}(x)=\left(\begin{array}{c|c}
1 & 0 \\
\hline 0 & A^{T}
\end{array}\right) \cdot K l(x) \cdot\left(\begin{array}{c|c}
1 & 0 \\
\hline 0 & A
\end{array}\right)
$$

so both sides of inequality (7) are invariant under volume preserving affine transformations.

The rest of the paper is organized as follows. In Section 2 we fix some notations and present some background in convex geometry to be used throughout the paper. In Section 3 we define the set $N_{p}$ and prove Theorem 1.5] and Corollary 1.6. In Section 4 we study the dual Busemann Random Simplex Inequality and prove Theorems 1.8 and 1.9. Finally in Section 5 we discuss the conjectured inequality (5), and its relation to the Petty conjecture.

This work was partly written during a short visit at TU Wien. We would like to thank professor F. Schuster and his team of collaborators for their hospitality, useful comments and fruitful discussions.

The author was partially supported by Fapemig, Project APQ-01542-18.

\section{Notation And Preliminaries}

This section is devoted to basic definitions and notations within the convex geometry. For a comprehensive reference in convex geometry we refer to the book [26].

We recall that a convex body $K \subset \mathbb{R}^{n}$ is a convex compact subset of $\mathbb{R}^{n}$ with non-empty interior.

The support function $h_{K}$ is defined as

$$
h_{K}(y)=\max \{\langle y, z\rangle / z \in K\},
$$

and uniquely characterizes $K$. If $K$ contains the origin in the interior, then we also have the gauge $\|\cdot\|_{K}$ and radial $r_{K}(\cdot)$ functions of $K$ defined respectively as

$$
\begin{gathered}
\|y\|_{K}:=\inf \{\lambda>0: y \in \lambda K\}, \quad y \in \mathbb{R}^{n} \backslash\{0\}, \\
r_{K}(y):=\max \{\lambda>0: \lambda y \in K\}, \quad y \in \mathbb{R}^{n} \backslash\{0\} .
\end{gathered}
$$

Clearly, $\|y\|_{K}=\frac{1}{r_{K}(y)}$. We also recall that $\|\cdot\|_{K}$ is actually a norm when the convex body $K$ is centrally symmetric, i.e. $K=-K$, and the unit ball with respect to $\|\cdot\|_{K}$ is just $K$. On the other hand, a general norm on $\mathbb{R}^{n}$ is uniquely determined by its unit ball, which is a centrally symmetric convex body.

For a convex body $K \subset \mathbb{R}^{n}$ containing the origin in its interior we define the polar body, denoted by $K^{\circ}$, by

$$
K^{\circ}:=\left\{y \in \mathbb{R}^{n} /\langle y, z\rangle \leq 1 \quad \forall z \in K\right\} .
$$

Evidently, $h_{K}^{-1}=r_{K^{\circ}}$. 
For $p \geq 1$, the $L_{p}$-mixed volume $V_{p}(K, L)$ of convex bodies $K$ and $L$ is defined by

$$
V_{p}(K, L)=\frac{p}{n} \lim _{\varepsilon \rightarrow 0} \frac{\operatorname{vol}\left(K+{ }_{p} \varepsilon \cdot{ }_{p} L\right)-\operatorname{vol}(K)}{\varepsilon},
$$

where $K+{ }_{p} \varepsilon \cdot{ }_{p} L$ is the convex body defined by:

$$
h_{K+{ }_{p} \varepsilon \cdot{ }_{p} L}(x)^{p}=h_{K}(x)^{p}+\varepsilon h_{L}(x)^{p}, \quad \forall x \in \mathbb{R}^{n} .
$$

It is known (see [15]) that there exists a unique finite positive Borel measure $S_{p}(K,$.$) on \mathbb{S}^{n-1}$ such that

$$
V_{p}(K, L)=\frac{1}{n} \int_{\mathbb{S}^{n-1}} h_{L}(u)^{p} d S_{p}(K, u)
$$

for each convex body $L$.

If $1 \leq p<\infty$ and $K, L$ are convex bodies in $\mathbb{R}^{n}$ containing the origin as interior point, we can find also in [15] that

$$
V_{p}(K, L) \geq \operatorname{vol}(K)^{\frac{n-p}{n}} \operatorname{vol}(L)^{\frac{p}{n}}
$$

with equality if and only if $K$ and $L$ are dilates of each other.

The $L^{p}$ surface area measure of a function $f: \mathbb{R}^{n} \rightarrow \mathbb{R}$ with $L^{p}$ weak derivative is given by the lemma:

Lemma 2.1 (Lemma 4.1 of [16]). Given $1 \leq p<\infty$ and a function $f: \mathbb{R}^{n} \rightarrow \mathbb{R}$ with $L^{p}$ weak derivative, there exists a unique finite Borel measure $S_{p}(f,$.$) on \mathbb{S}^{n-1}$ such that

$$
\int_{\mathbb{R}^{n}} \phi(-\nabla f(x))^{p} d x=\int_{\mathbb{S}^{n-1}} \phi(u)^{p} d S_{p}(f, u),
$$

for every non-negative continuous function $\phi: \mathbb{R}^{n} \rightarrow \mathbb{R}$ homogeneous of degree 1 .

Conversely, for a convex body $L$ the function $f_{L}(x)=F\left(\|x\|_{L}\right)$ satisfies $S_{p}(f,)=.S_{p}(L,$.$) if F$ is any function $F: \mathbb{R}_{+} \rightarrow \mathbb{R}_{+}$satisfying

$$
\int_{0}^{\infty} t^{n-1} F^{\prime}(t)^{p} d t=1
$$

(see [16, Formula (4.13) ]). In view of identity (요 ), for any $f$ and $L$ such that $S_{p}(f,)=.S_{p}(L,$.$) , we have$

$$
V_{p}(L, K)=\frac{1}{n} \int_{\mathbb{R}^{n}} h(K,-\nabla f(x))^{p} d x .
$$

This allows us to define the functional mixed volume. For $f$ a continuous non-negative function with compact support we define

$$
\begin{aligned}
V_{p}(f, K) & =\frac{1}{n} \int_{S^{n-1}} h(K, \xi)^{p} d S_{p}(f, \xi) \\
& =\frac{1}{n} \int_{\mathbb{R}^{n}} h(K,-\nabla f(x))^{p} d x .
\end{aligned}
$$

The $L_{p}$ Sharp Sobolev inequality for general norms was proved by Cordero, Nazaret and Villani in [6] using a mass-transportation method, and it extends the mixed volume inequality to the functional setting. If $1 \leq p<n, L$ is a centrally symmetric convex body and $f$ is a continuous non-negative function with compact support, we have

$$
V_{p}(f, L)=\frac{1}{n} \int_{\mathbb{R}^{n}}\|\nabla f(x)\|_{L^{\circ}}^{p} d x \geq \operatorname{cnv}_{n, p}\|f\|_{p^{*}}^{p} \operatorname{vol}(L)^{p / n}
$$


where equality holds if and only if $f(x)=a F_{p}\left(b\left\|x-x_{0}\right\|_{L}\right)$ with $x_{0} \in \mathbb{R}^{n}, a, b>0$ and

$$
F_{p}(t)=\left\{\begin{array}{c}
\left(1+t^{\frac{p}{p-1}}\right)^{1-\frac{n}{p}} \text { if } p \in(1, n) \\
\chi_{[0,1]}(t) \text { if } p=1
\end{array}\right.
$$

For $p=1$ the equality is understood in the weak sense. For $L=B_{2}$ we recover the euclidean $L_{p^{-}}$Sobolev inequality

$$
\mathcal{S}_{n, p}\left\||\nabla f(x)|_{2}\right\|_{L^{p}} \geq\|f\|_{p^{*}}
$$

with constant $\mathcal{S}_{n, p}=\left(n \operatorname{cnv}_{n, p} \omega_{n}^{\frac{p}{n}}\right)^{-1 / p}$ where $\omega_{n}$ is the volume of the unit euclidean ball. We refer to [12, equation (5)] for the precise value of $\mathcal{S}_{n, p}$.

Definition 2.2. For a convex body $K$ and $1 \leq p<n$ we define the function $F_{p, K}(x)=a F_{p}\left(\|x\|_{K}\right)$ where a is defined by the requirement that

$$
V_{p}\left(F_{p, K}, L\right)=V_{p}(K, L)
$$

for every convex body $L$.

Inserting $F_{p, L}$ in (9) and using the equality case, we have

$$
\operatorname{cnv}_{n, p}\left\|F_{p, K}\right\|_{p^{*}}^{p}=\operatorname{vol}(K)^{\frac{n-p}{n}} .
$$

For $p \geq 1$, the $L_{p}$-dual mixed volume $\tilde{V}_{-p}(K, L)$ of two bodies $K, L$ is defined by

$$
\tilde{V}_{-p}(K, L)=\frac{n+p}{n} \int_{K}\|x\|_{L}^{p} d x
$$

and the $L_{p}$-dual mixed volume inequality states that

$$
\tilde{V}_{-p}(K, L) \geq \operatorname{vol}(K)^{\frac{n+p}{n}} \operatorname{vol}(L)^{-\frac{p}{n}} .
$$

Definition (10) extends easily to functions (see [18, equation (2.6)]) as

$$
\tilde{V}_{-p}(f, L)=\frac{n+p}{n} \int_{\mathbb{R}^{n}} f(x)\|x\|_{L}^{p} d x .
$$

For a convex body $K$, the function $f_{K}(x)=F\left(\|x\|_{K}\right)$ satisfies $\tilde{V}_{-p}(f, L)=\tilde{V}_{-p}(K, L)$ for every convex body $L$, provided that the function $F$ satisfies

$$
(n+p) \int_{0}^{\infty} t^{n+p-1} F(t) d t=1
$$

The next functional inequality is an extension to functions of the $L_{p}$-dual mixed volume inequality.

Lemma 2.3 ([18, Lemma 4.1]). Let $\lambda \in\left(\frac{n}{n+p}, 1\right) \cup(1,+\infty]$ and $L$ be a convex body with the origin in its interior. Then, for any non-zero non-negative continuous function $f: \mathbb{R}^{n} \rightarrow \mathbb{R}$ with compact support,

$$
\tilde{V}_{-p}(f, L) \geq m_{n, p, \lambda}\|f\|_{1}^{\frac{n+p \lambda^{\prime}}{n}}\|f\|_{\lambda}^{-\frac{p}{n} \lambda^{\prime}} \operatorname{vol}(L)^{-\frac{p}{n}} .
$$

We refer to [18, Lemma 4.1] for the explicit value of the sharp constant $m_{n, p, \lambda}$. Moreover, equality holds if and only if $f(x)=a G_{p, \lambda}\left(b\|x\|_{L}\right)$ for constants $a, b \in \mathbb{R}$.

Definition 2.4. For a convex body $K$ we define the function $G_{p, \lambda, K}(x)=a G_{p, \lambda}\left(\|x\|_{K}\right)$ where a is defined by the requirement that $\tilde{V}_{-p}\left(G_{p, \lambda, K}, L\right)=\tilde{V}_{-p}(K, L)$ for every convex body $L$. 
Inserting $G_{p, \lambda, L}$ in (9) and using the equality case, we obtain

$$
m_{n, p, \lambda}\left\|G_{p, \lambda, L}\right\|_{1}^{\frac{n+p \lambda^{\prime}}{n}}\left\|G_{p, \lambda, L}\right\|_{\lambda}^{-\frac{p}{n} \lambda^{\prime}}=\operatorname{vol}(L)^{\frac{n+p}{n}} .
$$

It is easy to see how the $L_{p}$-dual mixed volume relates to the $L_{p}$ Random Simplex Inequality, as

$$
\tilde{V}_{-p}\left(L_{n}, N_{p}^{\circ}\left(L_{1}, \ldots, L_{n-1}\right)\right)=\frac{n+p}{n} I_{p}\left(L_{1}, \ldots, L_{n}\right) .
$$

We also have $I_{p}\left(G_{p, \lambda, L_{1}}, \ldots, G_{p, \lambda, L_{n}}\right)=I_{p}\left(L_{1}, \ldots, L_{n}\right)$ and $\tilde{I}_{p}\left(F_{p, L_{1}}, \ldots, F_{p, L_{n}}\right)=\tilde{I}_{p}\left(L_{1}, \ldots, L_{n}\right)$.

For a convex body $K$ with smooth boundary and strictly positive Gauss curvature, the $L_{p}$ surface area measure of $K$ is absolutely continuous with respect to the invariant measure of the sphere and

$$
\int_{S^{n-1}} \phi(\xi) d S_{p, K}(\xi)=\int_{S^{n-1}} \phi(\xi) f_{p}(K, \xi) d \xi
$$

for every measurable function $\phi$, where $f_{p}$ is called the $L_{p}$ curvature function. For $p=1$ this is the inverse of the Gauss curvature. The $p$-affine surface area of $K$ is defined by

$$
\begin{aligned}
\Omega_{p}(K) & =\int_{S^{n-1}} f_{p}(K, \xi)^{\frac{n}{n+p}} d \xi \\
& =\int_{S^{n-1}} f_{p}(K, \xi)^{-\frac{p}{n+p}} d S_{p, K} .
\end{aligned}
$$

For $p=1$ we obtain the affine surface area measure

$$
\Omega(K)=\int_{\partial K} \kappa(x)^{\frac{1}{n+1}} d x .
$$

We refer to [26, Section 10.5] for more general definitions, and to [20] for a geometric interpretation. $\Omega_{p}$ is a centro-affine invariant (invariant under volume preserving linear transformations), but not translation invariant unless $p=1$.

\section{Functional Random Simplex Inequality}

Given functions $l_{1}, \ldots, l_{k}$ and convex bodies $L_{k+1}, \ldots, L_{n}$, we write $I_{p}\left(l_{1}, \ldots, l_{k}, L_{k+1}, \ldots, L_{n}\right)=I_{p}\left(l_{1}, \ldots, l_{n}\right)$ where $l_{i}=G_{p, \lambda, L_{i}}$ for $i=k+1, \ldots, n$. This quantity is independent of $\lambda$.

\section{Proof of Theorem 1.5:}

We prove by induction in $k$, the following

Claim. Let $l_{1}, \ldots, l_{k}$ be continuous non-negative functions with compact support, and let $L_{k+1}, \ldots, L_{n-1}$ be convex bodies, then

$$
\operatorname{vol}\left(N_{p}^{\circ}\left(l_{1}, \ldots, l_{k}, L_{k+1}, \ldots, L_{n-1}\right)\right) \leq a_{n, p} \prod_{i=1}^{k} m_{n, p, \lambda}^{-\frac{n}{p}}\left\|l_{i}\right\|_{1}^{-\frac{n+p \lambda^{\prime}}{p}}\left\|l_{i}\right\|_{\lambda}^{\lambda^{\prime}} \prod_{j=k+1}^{n-1} \operatorname{vol}\left(L_{j}\right)^{-\frac{n+p}{p}}
$$

and equality holds if and only if $l_{1}, \ldots, l_{k}$ have the form $l_{i}(x)=a_{i} F_{p}\left(b_{i}\left|A .\left(x-x_{i}\right)\right|_{2}\right)$ where $x_{i} \in \mathbb{R}^{n}, A \in$ $\mathrm{Gl}_{n}(\mathbb{R})$, and $L_{i}=a_{i} A^{-1} \cdot B_{2}$ for $i \geq k+1$. Taking $k=n-1$ we obtain the Theorem with $A_{n, p, \lambda}=a_{n, p} m_{n, p, \lambda}^{-\frac{n^{2}}{p}}$.

For $k=0$ the claim is exactly Theorem 1.3. Assume $k \geq 1$ and that the Lemma is true with $k$ replaced by $k-1$. Given functions $l_{1}, \ldots, l_{k}$ and sets $L_{k+1}, \ldots, L_{n-1}$ take

$$
K=N_{p}^{\circ}\left(l_{1}, \ldots, l_{k-1}, N_{p}^{\circ}\left(l_{1}, \ldots, l_{k}, L_{k+1}, \ldots, L_{n-1}\right), L_{k+1}, \ldots, L_{n-1}\right) .
$$


Using the commutativity of $I_{p}$,

$$
\begin{aligned}
\tilde{V}_{-p}\left(l_{k}, K\right) & =\frac{n+p}{n} I_{p}\left(l_{1}, \ldots, l_{k-1}, N_{p}^{\circ}\left(l_{1}, \ldots, l_{k}, L_{k+1}, \ldots, L_{n-1}\right), L_{k+1}, \ldots, L_{n-1}, l_{k}\right) \\
& =\frac{n+p}{n} I_{p}\left(l_{1}, \ldots, l_{k-1}, l_{k}, L_{k+1}, \ldots, L_{n-1}, N_{p}^{\circ}\left(l_{1}, \ldots, l_{k}, L_{k+1}, \ldots, L_{n-1}\right)\right) \\
& =\tilde{V}_{-p}\left(N_{p}^{\circ}\left(l_{1}, \ldots, l_{k}, L_{k+1}, \ldots, L_{n-1}\right), N_{p}^{\circ}\left(l_{1}, \ldots, l_{k}, L_{k+1}, \ldots, L_{n-1}\right)\right) \\
& =\operatorname{vol}\left(N_{p}^{\circ}\left(l_{1}, \ldots, l_{k}, L_{k+1}, \ldots, L_{n-1}\right)\right)
\end{aligned}
$$

then by (11)

$$
\operatorname{vol}\left(N_{p}^{\circ}\left(l_{1}, \ldots, l_{k}, L_{k+1}, \ldots, L_{n-1}\right)\right) \geq m_{n, p, \lambda}\left\|l_{k}\right\|_{1}^{\frac{n+p \lambda^{\prime}}{n}}\left\|l_{k}\right\|_{\lambda}^{-\frac{p \lambda^{\prime}}{n}} \operatorname{vol}(K)^{-\frac{p}{n}} .
$$

Notice that the definition of $K$ involves only $k-1$ functions. Then by the induction hypothesis we have

$$
\begin{aligned}
& \operatorname{vol}\left(N_{p}^{\circ}\left(l_{1}, \ldots, l_{k}, L_{k+1}, \ldots, L_{n-1}\right)\right) \geq m_{n, p, \lambda}\left\|l_{k}\right\|_{1}^{\frac{n+p \lambda^{\prime}}{n}}\left\|l_{k}\right\|_{\lambda}^{-\frac{p \lambda^{\prime}}{n}} \\
& \times\left(a_{n, p} \prod_{i=1}^{k-1} m_{n, p, \lambda}^{-\frac{n}{p}}\left\|l_{i}\right\|_{1}^{-\frac{n+p \lambda^{\prime}}{p}}\left\|l_{i}\right\|_{\lambda}^{\frac{p \lambda^{\prime}}{p}} \operatorname{vol}\left(N_{p}^{\circ}\left(l_{1}, \ldots, l_{k}, L_{k+1}, \ldots, L_{n-1}\right)\right)^{-\frac{n+p}{p}} \prod_{j=k+1}^{n-1} \operatorname{vol}\left(L_{j}\right)^{-\frac{n+p}{p}}\right)^{-\frac{p}{n}} \\
&=m_{n, p, \lambda}\left\|l_{k}\right\|_{1}^{\frac{n+p \lambda^{\prime}}{n}}\left\|l_{k}\right\|_{\lambda}^{-\frac{p \lambda^{\prime}}{n}} \\
& \times\left(a_{n, p} \prod_{i=1}^{k-1} m_{n, p, \lambda}^{-\frac{n}{p}}\left\|l_{i}\right\|_{1}^{-\frac{n+p \lambda^{\prime}}{p}}\left\|l_{i}\right\|_{\lambda}^{\lambda^{\prime}} \prod_{j=k+1}^{n-1} \operatorname{vol}\left(L_{j}\right)^{-\frac{n+p}{p}}\right)^{-\frac{p}{n}} \operatorname{vol}\left(N_{p}^{\circ}\left(l_{1}, \ldots, l_{k}, L_{k+1}, \ldots, L_{n-1}\right)\right)^{\frac{n+p}{n}}
\end{aligned}
$$

that proves the Lemma.

The equality case follows from the equality case of the induction hypothesis (the equality case of Theorem 1.3 for $k=n-1$ ), and the equality case of (11).

We complete the proof of Corollary 1.6 by proving the equivalence between random and isoperimetric inequalities in the functional setting.

Proof of Corollary 1.6.

By the dual mixed volume inequality and Theorem 1.5.

$$
\begin{aligned}
I_{p}\left(l_{1}, \ldots, l_{n}\right) & =\frac{n}{n+p} \tilde{V}_{-p}\left(l_{n}, N_{p}^{\circ}\left(l_{1}, \ldots, l_{n-1}\right)\right) \\
& \geq \frac{n}{n+p} m_{n, p, \lambda}\left\|l_{n}\right\|_{1}^{\frac{n+p \lambda^{\prime}}{n}}\left\|l_{n}\right\|_{\lambda}^{-\frac{p \lambda^{\prime}}{n}} \operatorname{vol}\left(N_{p}^{\circ}\left(l_{1}, \ldots, l_{n-1}\right)\right)^{-\frac{p}{n}} \\
& \geq B_{n, p, \lambda}\left\|l_{n}\right\|_{1}^{\frac{n+p \lambda^{\prime}}{n}}\left\|l_{n}\right\|_{\lambda}^{-\frac{p \lambda^{\prime}}{n}} \prod_{i=1}^{n-1}\left\|l_{i}\right\|_{1}^{\frac{n+p \lambda^{\prime}}{n}}\left\|l_{i}\right\|_{\lambda}^{-\frac{p \lambda^{\prime}}{n}}
\end{aligned}
$$

where $B_{n, p, \lambda}=\frac{n}{n+p} m_{n, p, \lambda} A_{n, p, \lambda}^{-\frac{p}{n}}$.

\section{The Dual Random Simplex Inequality}

In this section we prove Theorems 1.8 and 1.9. Let $L$ be a convex body with smooth boundary and positive Gauss curvature, then it has a well defined $p$-curvature function $f_{p, L}$ defined as the density of the 
$p$-surface area measure with respect to the Lebesgue measure in the sphere, this is

$$
d S_{p, L}=f_{p, L}(\xi) d \xi .
$$

For $L$ as before, we consider the star body $L_{p}^{*}$ given by the radial function $r\left(L_{p}^{*}, \xi\right)=f_{p, L}(\xi)^{\frac{1}{n+p}}$ and its volume given by

$$
\operatorname{vol}\left(L_{p}^{*}\right)=\frac{1}{n} \int_{S^{n-1}} f_{p, L}(\xi)^{\frac{n}{n+p}}=\frac{1}{n} \Omega_{p}(L) .
$$

Now we prove Theorem 1.8 .

Proof of Theorem 1.8:

By definition of $\tilde{I}_{p}$ and $L_{p}^{*}$,

$$
\begin{aligned}
\tilde{I}_{p}\left(L_{1}, \ldots, L_{n}\right) & =\int_{S^{n-1}} \cdots \int_{S^{n-1}} D_{n}\left(\xi_{1}, \ldots, \xi_{n}\right)^{p} d S_{p, L_{1}}\left(\xi_{1}\right) \ldots d S_{p, L_{n}}\left(\xi_{n}\right) \\
& =\int_{S^{n-1}} \cdots \int_{S^{n-1}} D_{n}\left(\xi_{1}, \ldots, \xi_{n}\right)^{p} \prod_{i=1}^{n} f_{p, L_{i}}\left(\xi_{i}\right) d \xi_{1} \ldots d \xi_{n} \\
& =\int_{S^{n-1}} \cdots \int_{S^{n-1}} D_{n}\left(\xi_{1}, \ldots, \xi_{n}\right)^{p} \prod_{i=1}^{n} r_{\left(L_{i}\right)_{p}^{*}}\left(\xi_{i}\right)^{n+p} d \xi_{1} \ldots d \xi_{n} \\
& =(n+p)^{n} \int_{\left(L_{1}\right)_{p}^{*}} \ldots \int_{\left(L_{n}\right)_{p}^{*}} D_{n}\left(x_{1}, \ldots, x_{n}\right)^{p} d x_{1} \ldots d x_{n} \\
& =(n+p)^{n} I_{p}\left(\left(L_{1}\right)_{p}^{*}, \cdots,\left(L_{n}\right)_{p}^{*}\right)
\end{aligned}
$$

The sets $\left(L_{i}\right)_{p}^{*}$ are not necessarily convex, but Corollary 1.6 applied to indicator functions with $\lambda=\infty$ implies that Theorem 1.1 remains valid for measurable sets (notice that $m_{n, p, \infty}=1$ ). By this inequality and identity (12) we get

$$
\tilde{I}_{p}\left(L_{1}, \cdots, L_{n}\right) \geq(n+p)^{n} b_{n, p} \prod_{i=1}^{n} \operatorname{vol}\left(\left(L_{i}\right)_{p}^{*}\right)^{\frac{n+p}{n}}=(n+p)^{n} \frac{b_{n, p}}{n^{n+p}} \prod_{i=1}^{n} \Omega_{p}\left(L_{i}\right)^{\frac{n+p}{n}}
$$

The proof of Theorem 1.9 requires a simple lemma:

Lemma 4.1. Let $g:(0, \infty) \rightarrow[0, \infty)$ be a continuous non-negative function with compact support and $\lambda \in\left(\frac{n}{n+p}, 1\right) \cup(1,+\infty]$, then

$$
\left(\int_{0}^{\infty} g(t)^{\frac{n+p}{n}} d t\right)^{\frac{n}{n+p}} \geq L_{n, p, \lambda}\left(\int_{0}^{\infty} g(t) t^{\lambda-1} d t\right)^{-\frac{p}{n+p} \frac{1}{\lambda-1}}\left(\int_{0}^{\infty} g(t) d t\right)^{\frac{n+p \lambda^{\prime}}{n+p}}
$$

where

$$
L_{n, p, \lambda}=\left\{\begin{array}{cl}
(1-\lambda)\left(\frac{p}{n+p}\right)^{\frac{p}{(\lambda-1)(n+p)}\left(\lambda-\frac{n}{n+p}\right)^{\frac{n-\lambda(n+p)}{(\lambda-1)(n+p)}} \frac{\Gamma\left(\frac{1}{1-\lambda}\right)}{\Gamma\left(\frac{n}{p}+2\right) \Gamma\left(\frac{\lambda}{1-\lambda}-\frac{n}{p}\right)}} & \text { if } \lambda<1 \\
(\lambda-1)\left(\frac{p}{n+p}\right)^{\frac{p}{(\lambda-1)(n+p)}}\left(\lambda+\frac{p}{n+p}-1\right)^{\frac{-\lambda n+n-\lambda p}{(\lambda-1)(n+p)}} \frac{\Gamma\left(\frac{n}{p}+\frac{1}{\lambda-1}+2\right)}{\Gamma\left(\frac{\lambda}{\lambda-1}\right) \Gamma\left(\frac{n}{p}+2\right)} & \text { if } \lambda>1
\end{array}\right.
$$


and equality holds if and only if $g(t)=a p_{\lambda}(t / r)$ for some $a, r>0$ and

$$
p_{\lambda}(t)=\left\{\begin{array}{cc}
\left(t^{\lambda-1}-1\right)_{+}^{\frac{n}{p}} & \text { if } \lambda<1 \\
\left(1-t^{\lambda-1}\right)_{+}^{\frac{n}{p}} & \text { if } \lambda>1 \\
\chi_{[0,1]}(t) & \text { if } \lambda=\infty .
\end{array}\right.
$$

For $\lambda=\infty$ we interpret inequality (14) as

$$
\left(\int_{0}^{\infty} g(t)^{\frac{n+p}{n}} d t\right)^{\frac{n}{n+p}} \geq(\mathrm{S} g)^{-\frac{p}{n+p}} \int_{0}^{\infty} g(t) d t
$$

where $\mathrm{S} g$ is the minimum $a>0$ such that $g(t)=0$ for all $t>a$.

Proof. For $\lambda>1$ and $t>0$, let $p_{\lambda}(t)=\left(1-t^{\lambda-1}\right)_{+}^{\frac{n}{p}}$. Then

$$
p_{\lambda}(t / r)^{\frac{p}{n}} \geq 1-t^{\lambda-1} r^{1-\lambda} .
$$

Multiplying by $g(t)$ and integrating

$$
\int_{0}^{\infty} g(t) p_{\lambda}(t / r)^{\frac{p}{n}} d t \geq \int_{0}^{\infty} g(t) d t-r^{1-\lambda} \int_{0}^{\infty} g(t) t^{\lambda-1} d t
$$

By Hölder,

$$
\begin{aligned}
\int_{0}^{\infty} g(t) d t & \leq \int_{0}^{\infty} g(t) p_{\lambda}(t / r)^{\frac{p}{n}} d t+r^{1-\lambda} \int_{0}^{\infty} g(t) t^{\lambda-1} d t \\
& \leq\left(\int_{0}^{\infty} g(t)^{\frac{n+p}{n}} d t\right)^{\frac{n}{n+p}}\left(\int_{0}^{\infty} p_{\lambda}(t / r)^{\frac{n+p}{n}} d t\right)^{\frac{p}{n+p}}+r^{1-\lambda} \int_{0}^{\infty} g(t) t^{\lambda-1} d t \\
& \leq\left(\int_{0}^{\infty} g(t)^{\frac{n+p}{n}} d t\right)^{\frac{n}{n+p}}\left(\int_{0}^{\infty} p_{\lambda}(t)^{\frac{n+p}{n}} d t\right)^{\frac{p}{n+p}} r^{\frac{p}{n+p}}+r^{1-\lambda} \int_{0}^{\infty} g(t) t^{\lambda-1} d t .
\end{aligned}
$$

Minimizing the right-hand side for $r>0$, we obtain the result for $\lambda>1$. For the case $\lambda \in\left(\frac{n}{n+p}, 1\right)$, we define $q_{\lambda}(t)=\left(t^{\lambda-1}-1\right)_{+}^{\frac{n}{p}}$. Then, $q_{\lambda}(t)^{\frac{p}{n}} \geq t^{\lambda-1}-1$ and it follows that

$$
\int_{0}^{\infty} g(t) q_{\lambda}(t / r)^{\frac{p}{n}} d t \geq r^{1-\lambda} \int_{0}^{\infty} g(t) t^{\lambda-1} d t-\int_{0}^{\infty} g(t) d t .
$$

By Hölder

$$
\begin{aligned}
\int_{0}^{\infty} g(t) t^{\lambda-1} d t & \leq r^{\lambda-1} \int_{0}^{\infty} g(t) d t+r^{\lambda-1}\left(\int_{0}^{\infty} g(t)^{\frac{n+p}{n}} d t\right)^{\frac{n}{n+p}}\left(\int_{0}^{\infty} q_{\lambda}(t / r)^{\frac{n+p}{n}} d t\right)^{\frac{p}{n+p}} \\
& \leq r^{\lambda-1} \int_{0}^{\infty} g(t) d t+r^{\lambda-\frac{n}{n+p}}\left(\int_{0}^{\infty} g(t)^{\frac{n+p}{n}} d t\right)^{\frac{n}{n+p}}\left(\int_{0}^{\infty} q_{\lambda}(t)^{\frac{n+p}{n}} d t\right)^{\frac{p}{n+p}}
\end{aligned}
$$

and again we conclude minimizing with respect to $r>0$. The case $\lambda=\infty$ is just an application of Hölder inequality.

\section{Proof of Theorem 1.9 :}

Let $l_{1}, \ldots, l_{n}$ be $C^{2}$ smooth, non-negative functions with compact support. By Sard's Lemma, almost every level set of each function $l_{i}$ is a $C^{2}$-smooth manifold. For such a function $l=l_{i}$, the relation (8) implies that the surface area measure of $l$ is absolutely continuous with respect to the invariant measure in $S^{n-1}$, then 
we also have a well defined $p$-curvature function $f_{p, l}$ such that $d S_{p, l}=f_{p, l}(\xi) d \xi$. We define the star body $(l)_{p}^{*}$ similarly, by

$$
r\left((l)_{p}^{*}, \xi\right)^{n+p}=f_{p, l}(\xi)
$$

As in (13) we compute

$$
\tilde{I}_{p}\left(l_{1}, \cdots, l_{n}\right)=I_{p}\left(\left(l_{1}\right)_{p}^{*}, \ldots,\left(l_{n}\right)_{p}^{*}\right) \geq b_{n, p} \prod_{i=1}^{n} \operatorname{vol}\left(\left(l_{i}\right)_{p}^{*}\right)^{\frac{n+p}{n}} .
$$

Fix $l=l_{i}$ for $i=1, \ldots n$. Consider the function $n^{l}: \mathbb{R}^{n} \rightarrow S^{n-1}$ given by $n^{l}(x)=\frac{\nabla l(x)}{|\nabla l(x)|_{2}}$. Again by Sard's Lemma applied to $n^{l}$, for almost every $\xi$, the set

$$
P(\xi)=\left\{x \in \mathbb{R}^{n} / \nabla l(x) \neq 0 \text { and } n^{l}(x)=\xi\right\}
$$

is a 1-dimensional submanifold of the open subset of $\mathbb{R}^{n}$ where $\nabla l \neq 0$. By the definition of $f_{p, l}$ we have for any $p$-homogeneous function $\phi$ on $\mathbb{R}^{n}$,

$$
\begin{aligned}
\int_{S^{n-1}} \phi(\xi) d S_{p, l} & =\int_{\mathbb{R}^{n}} \phi(\nabla l(x)) d x \\
& =\int_{S^{n-1}} \int_{P_{l}(\xi)} J_{n^{l}}(x)^{-1} \phi(\nabla l(x)) d x d \xi \\
& =\int_{S^{n-1}} \int_{P_{l}(\xi)} J_{n^{l}}(x)^{-1}|\nabla l(x)|^{p} d x \phi(\xi) d \xi
\end{aligned}
$$

where we used the generalised co-area formula [21], and $J_{n^{l}}$ is the Jacobian of the function $n^{l}: \mathbb{R}^{n} \rightarrow S^{n-1}$ given by $n^{l}(x)=\frac{\nabla l(x)}{|\nabla l(x)|_{2}}$. The Jacobian of a transformation at a point $x_{0}$ can be computed by the formula (see [21, Lemma 1.2])

$$
J=\frac{D_{n-1}\left(A \cdot u_{1}, \ldots, A \cdot u_{n-1}\right)\left|u_{n}\right|}{D_{n}\left(u_{1}, \ldots, u_{n-1}, u_{n}\right)}
$$

where $A$ is the differential of $n^{l}$ at $x_{0}, u_{n}$ is a vector generating the kernel of $A$ and $u_{1}, \ldots, u_{n-1}$ is any basis of a complementary subspace to $\operatorname{ker}(A)$. Now, taking $u_{1}, \ldots, u_{n-1}$ to be an orthonormal basis of the orthogonal space to $\xi$, and $u_{n}$ unitary, we obtain $J=\left|\kappa /\left\langle\xi, u_{n}\right\rangle\right|$ where $\kappa$ is the Gauss curvature of the level set of $l$ at $x_{0}$. Observe that $u_{n}$ is the unit tangent vector of the curve $P_{l}(\xi)$ at $x_{0}$. From (16) we obtain

$$
f_{p, l}(\xi)=\int_{P_{l}(\xi)} \kappa(x)^{-1}|\nabla l(x)|^{p}\left|\xi^{T}\right| d \mathcal{H}_{1}(x)
$$

where $\left|\xi^{T}\right|$ is the tangential component over the curve $P_{l}(\xi)$.

Since $l$ has convex level sets with positive Gauss curvature, each $P(\xi)$ can be parametrized by a curve $\gamma_{\xi}:\left(0,\|l\|_{\infty}\right) \rightarrow \mathbb{R}$ satisfying $l\left(\gamma_{\xi}(t)\right)=t$. We compute

$$
\begin{aligned}
f_{p, l}(\xi) & =\int_{0}^{\|l\|_{\infty}} \kappa\left(\gamma_{\xi}(t)\right)^{-1}\left|\nabla l\left(\gamma_{\xi}(t)\right)\right|^{p}\left|\left\langle\xi, \gamma_{\xi}^{\prime}(t)\right\rangle\right| d t \\
& =\int_{0}^{\|l\|_{\infty}} \kappa\left(\gamma_{\xi}(t)\right)^{-1}\left|\nabla l\left(\gamma_{\xi}(t)\right)\right|^{p-1}\left|\left\langle\nabla l\left(\gamma_{\xi}(t)\right), \gamma_{\xi}^{\prime}(t)\right\rangle\right| d t \\
& =\int_{0}^{\|l\|_{\infty}} \kappa\left(\gamma_{\xi}(t)\right)^{-1}\left|\nabla l\left(\gamma_{\xi}(t)\right)\right|^{p-1} d t .
\end{aligned}
$$


By formula (17), applying the parametrization given by $\gamma_{\xi}$, the Minkowski integral inequality and the definition of the surface area measure we have

$$
\begin{aligned}
\operatorname{vol}\left(l_{p}^{*}\right) & =\int_{S^{n-1}}\left(\int_{0}^{\|l\|_{\infty}} \kappa\left(\gamma_{\xi}(t)\right)^{-1}\left|\nabla l\left(\gamma_{\xi}(t)\right)\right|^{p-1} d t\right)^{\frac{n}{n+p}} d \xi \\
& \geq\left(\int_{0}^{\|l\|_{\infty}}\left(\int_{S^{n-1}} \kappa\left(\gamma_{\xi}(t)\right)^{-\frac{n}{n+p}}\left|\nabla l\left(\gamma_{\xi}(t)\right)\right|^{\frac{(p-1) n}{n+p}} d \xi\right)^{\frac{n+p}{n}} d t\right)^{\frac{n}{n+p}} \\
& =\left(\int_{0}^{\|l\|_{\infty}}\left(\int_{\partial N_{l, t}} \kappa(x)^{\frac{p}{n+p}}|\nabla l(x)|^{\frac{(p-1) n}{n+p}} d S_{N_{l, t}}(x)\right)^{\frac{n+p}{n}} d t\right)^{\frac{n}{n+p}} .
\end{aligned}
$$

The Gauss curvature of the level set of a function can be computed taking the differential of $n^{l}$ restricted to $\xi^{\perp}$ giving $\kappa=\operatorname{det}\left(\begin{array}{c|c}0 & \xi^{T} \\ \hline \xi & |\nabla l|^{-1} H l\end{array}\right)$, so we have $|\kappa|=|\operatorname{det}(K l)||\nabla l|^{-n-1}$ and

$$
\operatorname{vol}\left(l_{p}^{*}\right)=\left(\int_{0}^{\|l\|_{\infty}}\left(\int_{\partial N_{l, t}}|\operatorname{det}(K l(x))|^{\frac{p}{n+p}}|\nabla l(x)|^{-1} d S_{N_{l, t}}(x)\right)^{\frac{n+p}{n}} d t\right)^{\frac{n}{n+p}} .
$$

Let us define $\Omega_{p}(l, t)=\int_{\partial N_{l, t}}|\operatorname{det}(K l(x))|^{\frac{p}{n+p}}|\nabla l(x)|^{-1} d S_{N_{l, t}}(x)$, then a simple computation shows that

$$
\Omega_{p}\left(l^{\alpha}, t^{\alpha}\right)=\Omega_{p}(l, t)\left(\alpha t^{\alpha-1}\right)^{(p-1) \frac{n}{n+p}},
$$

and taking $\lambda=1+(\alpha-1)(n+1) \frac{p}{n+p}$ and $g(t)=\Omega_{p}(l, t)$ in Lemma 4.1,

$$
\begin{aligned}
\operatorname{vol}\left(l_{p}^{*}\right) & \geq\left(\int_{0}^{\infty} \Omega_{p}(l, t)^{\frac{n+p}{n}} d t\right)^{\frac{n}{n+p}} \\
& \geq L_{n, p, \lambda}\left(\int_{0}^{\infty} \Omega_{p}(l, t) d t\right)^{\frac{n+p \lambda^{\prime}}{n+p}}\left(\int_{0}^{\infty} \Omega_{p}(l, t) t^{\lambda-1} d t\right)^{-\frac{p}{(n+p)(\lambda-1)}} \\
& =\alpha^{\frac{p}{\lambda-1}} L_{n, p, \lambda}\left(\int_{0}^{\infty} \Omega_{p}(l, t) d t\right)^{\frac{n+p \lambda^{\prime}}{n+p}}\left(\int_{0}^{\infty} \Omega_{p}(l, t)\left(\alpha t^{\alpha-1}\right)^{(p-1) \frac{n}{n+p}}\left(\alpha t^{\alpha-1}\right) d t\right)^{-\frac{p}{(n+p)(\lambda-1)}} \\
& =\alpha^{\frac{p}{\lambda-1}} L_{n, p, \lambda}\left(\int_{0}^{\infty} \Omega_{p}(l, t) d t\right)^{\frac{n+\alpha^{\prime}}{n+1}}\left(\int_{0}^{\infty} \Omega_{p}\left(l^{\alpha}, s\right) d s\right)^{-\frac{1}{n+1} \frac{1}{\alpha-1}} \\
& =\alpha^{\frac{p}{\lambda-1}} L_{n, p, \lambda} \Omega_{p}(l)^{\frac{n+\alpha^{\prime}}{n+1}} \Omega_{p}\left(l^{\alpha}\right)^{-\frac{1}{n+1} \frac{1}{\alpha-1}}
\end{aligned}
$$

and we obtain the result with $\tilde{B}_{n, p, \alpha}=b_{n, p}\left(\frac{\alpha \frac{p}{\lambda-1}}{n} L_{n, p, \lambda}\right)^{n+p}$.

The equality case follows from the equality case of Theorem 1.1] in (15), the equality case of Lemma 4.1 for the function $\Omega_{p}(l, t)$, and the formula $\Omega_{p}\left(F\left(|x|_{2}\right), F(t)\right)=n \omega_{n} F^{\prime}(t)^{(p-1) \frac{n}{n+p}}$. 


\section{Open PROBLEMS}

In this section we discuss the dual inequality (5) and its relation to the body $\tilde{N}_{p}$ and the Petty conjecture. Let us consider the following open problem:

Problem 1. Let $L$ be a convex body and $1 \leq p<n$, then

$$
\tilde{I}_{p}\left(L_{1}, \ldots, L_{n}\right) \geq \bar{b}_{n, p} \prod_{i=1}^{n} \operatorname{vol}\left(L_{i}\right)^{\frac{n-p}{n}}
$$

and equality holds if and only if $L_{1}, \ldots, L_{n}$ are homothetic origin-symmetric ellipsoids.

Now we show that the proof of Theorem 1.5 works for $\tilde{I}_{p}$ and $\tilde{N}_{p}$ using the mixed volume. As explained in the introduction, inequality (5) is equivalent to the isoperimetric inequality $\operatorname{vol}\left(\tilde{N}_{p}\left(L_{1}, \ldots, L_{n-1}\right)\right) \geq$ $\bar{a}_{n, p} \prod_{i=1}^{n-1} \operatorname{vol}\left(L_{i}\right)^{\frac{n-p}{p}}$. The proof of this equivalence is similar.

Lemma 5.1. Assume Problem 1 holds. Let $l_{1}, \ldots, l_{n-1}$ be continuous non-negative functions with compact support, let $L_{k+1}, \ldots, L_{n-1}$ be convex bodies and $1 \leq p<n$, then

$$
\operatorname{vol}\left(\tilde{N}_{p}\left(l_{1}, \ldots, l_{k}, L_{k+1}, \ldots, L_{n-1}\right)\right) \geq \bar{a}_{n, p} \prod_{i=1}^{k} \operatorname{cnv}_{n, p}^{\frac{n}{p}}\left\|l_{i}\right\|_{p^{*}}^{n} \prod_{j=k+1}^{n-1} \operatorname{vol}\left(L_{j}\right)^{\frac{n-p}{p}}
$$

and equality holds if and only if $l_{1}, \ldots, l_{k}$ have the form $l_{i}(x)=a_{i} F_{p}\left(b_{i}\left|A .\left(x-x_{i}\right)\right|_{2}\right)$ where $x_{0} \in \mathbb{R}^{n}$, $A \in \mathrm{Gl}_{n}(\mathbb{R})$, and $L_{i}=a_{i} A^{-1} . B_{2}$ for $i \geq k+1$.

Proof. Take $K=\tilde{N}_{p}\left(l_{1}, \ldots, l_{k-1}, \tilde{N}_{p}\left(l_{1}, \ldots, l_{k-1}, l_{k}, L_{k+1}, \ldots, L_{n-1}\right), L_{k+1}, \ldots, L_{n-1}\right)$. As in the proof of Lemma 1.5,

$$
V_{p}\left(l_{k}, K\right)=\operatorname{vol}\left(\tilde{N}_{p}\left(l_{1}, \ldots, l_{k}, L_{k+1}, \ldots, L_{n-1}\right)\right)
$$

then by the functional mixed volume inequality (9) and the induction hypothesis we get

$$
\begin{aligned}
& \operatorname{vol}\left(\tilde{N}_{p}\left(l_{1}, \ldots, l_{k}, L_{k+1}, \ldots, L_{n-1}\right)\right) \geq \operatorname{cnv}_{n, p} \operatorname{vol}(K)^{\frac{p}{n}}\left\|l_{k}\right\|_{p^{*}}^{p} \\
& \quad \geq \operatorname{cnv}_{n, p}\left(\bar{a}_{n, p} \prod_{i=1}^{k-1} \operatorname{cnv}_{n, p}^{\frac{n}{p}}\left\|l_{i}\right\|_{p^{*}}^{n} \operatorname{vol}\left(N_{p}\left(l_{1}, \ldots, l_{k}, L_{k+1}, \ldots, L_{n-1}\right)\right)^{\frac{n-p}{p}} \prod_{j=k+1}^{n-1} \operatorname{vol}\left(L_{j}\right)^{\frac{n-p}{p}}\right)^{\frac{p}{n}}\left\|l_{k}\right\|_{p^{*}}^{p}
\end{aligned}
$$

and that proves the lemma. The equality case follows from the equality case of the induction hypothesis (the equality case of Problem 1 for $k=n-1$ ), and the equality case of (9).

Summarizing, Problem 1 is equivalent to the following sharp inequalities

$$
\begin{gathered}
\tilde{I}_{p}\left(l_{1}, \ldots, l_{n}\right) \geq \bar{B}_{n, p} \prod_{i=1}^{n}\left\|l_{i}\right\|_{p^{*}}^{p} \\
\operatorname{vol}\left(\tilde{N}_{p}\left(L_{1}, \ldots, L_{n-1}\right)\right) \geq \bar{a}_{n, p} \prod_{i=1}^{n-1} \operatorname{vol}\left(L_{i}\right)^{\frac{n-p}{p}} \\
\operatorname{vol}\left(\tilde{N}_{p}\left(l_{1}, \ldots, l_{n-1}\right)\right) \geq \bar{A}_{n, p} \prod_{i=1}^{n}\left\|l_{i}\right\|_{p^{*}}^{n}
\end{gathered}
$$


and equality holds if and only if $L_{1}, \ldots, L_{n}$ are homothetic origin-symmetric ellipsoids and $l_{1}, \ldots, l_{n}$ have the form $l_{i}(x)=a_{i} F_{p}\left(b_{i}\left|A .\left(x-x_{i}\right)\right|\right)$ with $x_{0} \in \mathbb{R}^{n}, a_{i}, b_{i}>0, A \in \mathrm{Gl}_{n}(\mathbb{R})$. Here the constants satisfy $\bar{b}_{n, p}=n \bar{a}_{n, p}^{p / n}, \bar{B}_{n, p}=n \operatorname{cnv}_{n, p} \bar{A}_{n, p}^{p / n}, \bar{A}_{n, p}=\bar{a}_{n, p} \operatorname{cnv}_{n, p}^{(n-1) \frac{n}{p}}$. Inequalities (18) and (20) are equivalent, as well as inequalities (19) and (21). Also by Lemma 5.1, (20) implies (21), while the converse is obvious.

As a motivation to these problems we make two remarks, first notice that a particular case of inequality (19) is a sharp, affine invariant, $L_{p}$ Sobolev-like inequality

$$
\left(\int_{\mathbb{R}^{n}} \cdots \int_{\mathbb{R}^{n}} D_{n}\left(\nabla f\left(x_{1}\right), \ldots, \nabla f\left(x_{n}\right)\right)^{p} d x_{1} \cdots d x_{n}\right)^{\frac{1}{n p}} \geq \bar{B}_{n, p}^{\frac{1}{n p}}\|f\|_{p^{*}} .
$$

Second, for $p=1$, we have $\frac{1}{n !} \tilde{I}_{1}(L, \ldots, L)=\operatorname{vol}(\Pi L)$, and inequality (18) for $L_{1}=\cdots=L_{n}=L$ becomes Petty's conjectured inequality (6). By the Blaschke-Santaló inequality for symmetric bodies [26, (10.28)], inequality (6) is stronger than the Petty Projection inequality

$$
\operatorname{vol}\left(\Pi^{\circ} L\right)^{-\frac{1}{n}} \geq \frac{\omega_{n-1}}{\omega_{n}} \operatorname{vol}(L)^{\frac{n-1}{n}} .
$$

Zhang proved in [28] a functional version of (23) that results in a Sharp Affine Sobolev inequality

$$
\left(\frac{1}{n} \int_{S^{n-1}}\left(\frac{1}{2} \int_{\mathbb{R}^{n}}|\langle\nabla f(x), \xi\rangle| d x\right)^{-n} d \xi\right)^{-\frac{1}{n}} \geq \frac{\omega_{n-1}}{\omega_{n}}\|f\|_{\frac{n}{n-1}}
$$

where the left-hand side is $\operatorname{vol}\left(\Pi^{\circ} K_{f}\right)^{-\frac{1}{n}}$ and $K_{f}$ is such that $S\left(K_{f}, \cdot\right)=S(f, \cdot)$. Again by the BlaschkeSantaló inequality applied to $\Pi K_{f}$ we obtain

$$
\left(\frac{1}{n} \int_{S^{n-1}}\left(\frac{1}{2} \int_{\mathbb{R}^{n}}|\langle\nabla f(x), \xi\rangle| d x\right)^{-n} d \xi\right)^{-\frac{1}{n}} \geq\left(\frac{1}{\omega_{n}^{2} n !} \int_{\mathbb{R}^{n}} \ldots \int_{\mathbb{R}^{n}} D_{n}\left(\nabla f\left(x_{1}\right), \ldots, \nabla f\left(x_{n}\right)\right) d x_{1} \cdots d x_{n}\right)^{\frac{1}{n}}
$$

meaning that the Sobolev-like inequality (22) for $p=1$ is stronger and directly implies the affine Sobolev inequality of Zhang (24). One can check that the value of $\bar{B}_{n, 1}^{1 / n}$ in (22) is consistent with inequality (24). Notice that any purely analytic proof of (25) must contour (or re-prove) the Blaschke-Santaló inequality. In any case, that doesn't seem a trivial thing to do, but it might extend to the $L_{p}$ case:

\section{Problem 2.}

$$
\left(\int_{S^{n-1}}\left(\int_{\mathbb{R}^{n}}|\langle\nabla f(x), \xi\rangle|^{p} d x\right)^{-\frac{n}{p}} d \xi\right)^{-\frac{1}{n}} \geq c_{n, p}\left(\int_{\mathbb{R}^{n}} \ldots \int_{\mathbb{R}^{n}} D_{n}\left(\nabla f\left(x_{1}\right), \ldots, \nabla f\left(x_{n}\right)\right)^{p} d x_{1} \cdots d x_{n}\right)^{\frac{1}{n p}}
$$

Lastly, to this moment we don't know whether Problem 1 for $p>1$ can be reduced to the particular case where $L_{1}=\cdots=L_{n}=L$. We believe these problems are worth to consider since they might shed some light into an important open problem that resisted the symmetrization approach.

\section{REFERENCES}

[1] J Bourgain, M Meyer, V Milman, and A Pajor. On a geometric inequality. In Geometric Aspects of Functional Analysis, pages 271-282. Springer, 1988.

[2] Herbert Busemann. Volume in terms of concurrent cross-sections. Pacific Journal of Mathematics, 3(1):1-12, 1953.

[3] Umut Caglar, Matthieu Fradelizi, Olivier Guédon, Joseph Lehec, Carsten Schütt, and Elisabeth M Werner. Functional versions of lp-affine surface area and entropy inequalities. International Mathematics Research Notices, 2016(4):1223-1250, 2015 . 
[4] S Campi and P Gronchi. Extremal convex sets for sylvester-busemann type functionals. Applicable Analysis, 85(1-3):129$141,2006$.

[5] Stefano Campi, Andrea Colesanti, and Paolo Gronchi. A note on sylvester's problem for random polytopes in a convex body. 1999.

[6] Dario Cordero-Erausquin, Bruno Nazaret, and Cédric Villani. A mass-transportation approach to sharp sobolev and gagliardo-nirenberg inequalities. Advances in Mathematics, 182(2):307-332, 2004.

[7] Susanna Dann, Grigoris Paouris, and Peter Pivovarov. Bounding marginal densities via affine isoperimetry. Proceedings of the London Mathematical Society, 113(2):140-162, 2016.

[8] Matthieu Fradelizi and Mathieu Meyer. Some functional forms of blaschke-santaló inequality. Mathematische Zeitschrift, 256(2):379-395, 2007.

[9] H Groemer. On some mean values associated with a randomly selected simplex in a convex set. Pacific Journal of Mathematics, 45(2):525-533, 1973.

[10] H Groemer. On the mean value of the volume of a random polytope in a convex set. Archiv der Mathematik, 25(1):86-90, 1974.

[11] Julian Haddad, C Hugo Jimenez, and Marcos Montenegro. Asymmetric blaschke-santaló functional inequalities. arXiv preprint arXiv:1810.02288, 2018.

[12] Julián Haddad, Carlos Hugo Jiménez, and Marcos Montenegro. Sharp affine sobolev type inequalities via the lp busemannpetty centroid inequality. Journal of Functional Analysis, 271(2):454-473, 2016.

[13] M Hartzoulaki and G Paouris. Quermassintegrals of a random polytope in a convex body. Archiv der Mathematik, 80(4):430$438,2003$.

[14] Erwin Lutwak. Mixed projection inequalities. Transactions of the American Mathematical Society, 287(1):91-105, 1985.

[15] Erwin Lutwak et al. The brunn-minkowski-firey theory. i. mixed volumes and the minkowski problem. J. Differential Geom, 38(1):131-150, 1993

[16] Erwin Lutwak, Deane Yang, and Gaoyong Zhang. Optimal sobolev norms and the lp minkowski problem. International Mathematics Research Notices, 2006, 2006.

[17] Erwin Lutwak, Deane Yang, Gaoyong Zhang, et al. Lp affine isoperimetric inequalities. J. Differential Geom, 56(1):111-132, 2000.

[18] Erwin Lutwak, Deane Yang, Gaoyong Zhang, et al. Moment-entropy inequalities. The Annals of Probability, 32(1B):757774, 2004.

[19] Erwin Lutwak, Gaoyong Zhang, et al. Blaschke-santaló inequalities. J. Differential Geom, 47(1):1-16, 1997.

[20] Mathieu Meyer and Elisabeth Werner. On the p-affine surface area. arXiv preprint math/9712276, 1997.

[21] Liviu I Nicolaescu. The coarea formula. In seminar notes. Citeseer, 2011.

[22] Grigoris Paouris and Peter Pivovarov. Randomized isoperimetric inequalities. In Convexity and Concentration, pages 391425. Springer, 2017.

[23] Clinton M Petty. Projection bodies. In Proc. Coll. Convexity, Copenhagen, pages 234-241, 1965.

[24] Richard E Pfiefer. Maximum and minimum sets for some geometric mean values. Journal of Theoretical Probability, 3(2):169-179, 1990.

[25] Christos Saroglou. Characterizations of extremals for some functionals on convex bodies. Canadian Journal of Mathematics, 62(6):1404-1418, 2010.

[26] Rolf Schneider. Convex bodies: the Brunn-Minkowski theory. Number 151. Cambridge university press, 2014.

[27] Rolf Schneider and Wolfgang Weil. Stochastic and integral geometry. Springer Science \& Business Media, 2008.

[28] Gaoyong Zhang. The affine sobolev inequality. J. Differential Geom, 53(1):183-202, 1999.

Julián Haddad: Departamento de Matemática, ICEx, Universidade Federal de Minas Gerais, 30.123-970, Belo Horizonte, Brasil.

E-mail address: jhaddad@mat.ufmg.br 